\title{
SOME PROBLEMS ABOUT THE REPRESENTATION OF MONOTONE OPERATORS BY CONVEX FUNCTIONS
}

\author{
JEAN-PAUL PENOT ${ }^{\bullet 1}$ and CONSTANTIN ZĂLINESCU ${ }^{2}$
}

Dedicated to the memory of Simon Fitzpatrick

(Received 30 July, 2004; revised 18 May, 2005)

\begin{abstract}
We answer a few questions raised by $S$. Fitzpatrick concerning the representation of maximal monotone operators by convex functions. We al so examine some other questions concerning this representation and other ones which have recently emerged.
\end{abstract}

\section{Introduction}

The interest of the theory of monotone operators does not arise just from the elegance of the results; it is also propelled by the number of applications, in particular for partial differential equations and variational inequalities (see $[1-3,7,10,24]$ and their bibliographies).

Several proposals have established links between maximal monotone operators and convex functions $([4-6,8,9,11,15,16,18,20])$. Some of these contributions reap advantage from these links in deriving the most important results of the theory of maximal monotone operators from known facts in convex analysis $([16,18-20])$. The richness of the theory of monotone operators which has given rise to a great number of works justifies an interest for these links. A first attempt along this line has been proposed in the book of Simons [18]; the pioneering proposal of Fitzpatrick in [6] and its recent avatars enable one to give a still simpler approach.

In the present paper we relate the different approaches mentioned above and we try to answer some questions raised by Fitzpatrick in [6]. We also deal with some

\footnotetext{
'Laboratoire de Mathématiques appliquées, ERS CNRS 2570, Faculté des sciences, av. de l'Université, 64000 PAU, France; e-mail: Jean-Paul.Penot@univ-pau.fr.

${ }^{2}$ University "Al. I. Cuza" laşi, Faculty of Mathematics, Bd. Carol I, Nr. 11, 700506 Iaşi, Romania; e-mail: zalinesc@uaic.ro.

(C) Australian Mathematical Society 2005, Serial-fee code 1446-1811/05
} 
other questions, such as characterising the images or the inverse images of the correspondences defined in $[6,12]$ and [16]. Moreover, in Section 3 we consider a general construction of maximal monotone operators and we provide a representative of the new operator in terms of representatives of the operators from which it is built. This construction can be specialised to sums and compositions. We show by a counterexample that the Fitzpatrick representative of the sum of two maximal monotone operators may be different from the natural candidate given as the lsc hull of the partial infimal convolution of the representative of the summands. We also give in the last section a formula yielding an auto-conjugate representative of a maximal monotone operator under an additional assumption.

We hope that the clarifications we bring will make these correspondences more usable and add to their lure.

\section{Correspondences between operators and functions}

Throughout the paper $X$ is a reflexive Banach space with dual $X^{*}$. For $f$ : $X \times X^{*} \rightarrow \overline{\mathbb{R}}:=\mathbb{R} \cup\{-\infty,+\infty\}$, the transpose $f^{\top}: X^{*} \times X \rightarrow \overline{\mathbb{R}}$ of $f$ is defined by $f^{\top}\left(x^{*}, x\right):=f\left(x, x^{*}\right)$; similarly for $g: X^{*} \times X \rightarrow \overline{\mathbb{B}}$ we set $g^{\top}\left(x, x^{*}\right):=g\left(x^{*}, x\right)$. Given a Banach space $Z$ and a function $h: Z \rightarrow \overline{\mathbb{R}}$, we say that $h$ is proper if $\operatorname{dom} h:=\{z \in Z \mid h(z)<+\infty\}$ is nonempty and if $h$ does not take the value $-\infty$. We use the conjugate $h^{*}$ of $h$ given by

$$
h^{*}\left(z^{*}\right):=\sup \left\{\left\langle z, z^{*}\right\rangle-h(z) \mid z \in Z\right\}
$$

and we set, for $z \in Z$ with $h(z) \in \mathbb{R}$,

$$
\partial h(z):=\left\{z^{*} \in Z^{*} \mid \forall w \in Z: h(w)-h(z) \geq\left\langle z^{*}, w-z\right\rangle\right\} ;
$$

$\partial h(z):=\emptyset$ for $h(z) \notin \mathbb{B}$. We often identify a multifunction $M: X \rightrightarrows X^{*}$ from $X$ to $X^{*}$ (also called an operator) with its graph gph $M:=\left\{\left(x, x^{*}\right) \in X \times X^{*} \mid x^{*} \in X^{*}\right\}$. Its domain is the set $\operatorname{dom} M:=\{x \in X \mid M(x) \neq \varnothing\}$.

To any function $f$ on $X \times X^{*}$ we associate the set

$$
T_{f}:=\left\{\left(x, x^{*}\right) \in X \times X^{*} \mid\left(x^{*}, x\right) \in \partial f\left(x, x^{*}\right)\right\}
$$

introduced by Fitzpatrick in [6] in the case when $f$ is convex; it is always a monotone subset of $X \times X^{*}$. Since here, as above, the subdifferential $\partial$ is taken in the sense of convex analysis, it is natural to take $f$ in the set $\Lambda\left(X \times X^{*}\right)$ of convex functions on $X \times X^{*}$ as Fitzpatrick did in [6]. The Fenchel equality enables us to reformulate the definition of $T_{f}$ as

$$
T_{f}=\left\{\left(x, x^{*}\right) \in X \times X^{*} \mid f\left(x, x^{*}\right)+f^{*}\left(x^{*}, x\right)=2\left\langle x, x^{*}\right\rangle\right\}
$$


Observe that if $T_{f}$ is nonempty then the lsc hull $\bar{f}$ of $f$ is proper (since then $f$ has a continuous affine minorant) and $T_{f} \subset T_{\bar{f}}$. So, if we are interested in obtaining a large $T_{f}$ (for example in order to get a maximal monotone multifunction), it is natural to take $f$ in the set $\Gamma\left(X \times X^{*}\right)$ of closed proper convex functions on $X \times X^{*}$. On the other hand, in several recent papers the classes

$$
\begin{aligned}
\mathscr{F} & :=\left\{f \in \Lambda\left(X \times X^{*}\right) \mid f \geq c\right\}, \\
\mathscr{G} & :=\left\{f \in \Gamma\left(X \times X^{*}\right) \mid f \geq c\right\}, \\
\mathscr{H} & :=\left\{f \in \Gamma\left(X \times X^{*}\right) \mid f \geq c, f^{*} \geq c^{\top}\right\},
\end{aligned}
$$

are considered, where $c: X \times X^{*} \rightarrow \mathbb{R}$ is the coupling function of the dual pair $\left(X, X^{*}\right)$, that is, $c\left(x, x^{*}\right):=\left\langle x, x^{*}\right\rangle:=x^{*}(x)$ for $x \in X$ and $x^{*} \in X^{*}$. To any $f \in \mathscr{F}$ one can associate the set

$$
M_{f}:=\left\{\left(x, x^{*}\right) \in X \times X^{*} \mid f\left(x, x^{*}\right)=c\left(x, x^{*}\right)\right\} .
$$

It has been shown in [16, Proposition 4] and [5, Theorem 3.1] that $M_{f}$ is monotone. In this case too, $M_{f} \subset M_{\bar{f}}$ since $c \leq \bar{f} \leq f$ and $\bar{f} \in \mathscr{G}$. So, also in this case, it is natural to take $f \in \Gamma\left(X \times X^{*}\right)$. Observe that for $f \in \Lambda\left(X \times X^{*}\right)$ one deduces from relation (2.1) that

$$
T_{f}=M_{8 f},
$$

where

$$
g_{f}\left(x, x^{*}\right)=\frac{1}{2} f\left(x, x^{*}\right)+\frac{1}{2} f^{*}\left(x^{*}, x\right) \quad \forall\left(x, x^{*}\right) \in X \times X^{*} ;
$$

moreover, the definition of the conjugate shows that $g_{f} \in \mathscr{F}$. Furthermore, Fitzpatrick [6, Theorem 2.4] proved that for $f \in \mathscr{F}$ one has $M_{f} \subset T_{f}$. This inclusion incites us to prefer $T_{f}$ to $M_{f}$; however the passage from $f$ to $M_{f}$ has some advantages, too.

Passages in the reverse direction are also of interest. To any nonempty subset $S \subset X \times X^{*}$ one can associate the function $\varphi_{S}$ on $X \times X^{*}$ given by

$$
\varphi_{S}\left(x, x^{*}\right):=\sup \left\{\left\langle x^{*}, w\right\rangle+\left\langle w^{*}, x-w\right\rangle \mid\left(w, w^{*}\right) \in S\right\},
$$

as Fitzpatrick did for the case when $S$ is the graph of a monotone operator ([6, Definition 3.1]). In [6, Theorem 3.4] Fitzpatrick showed that if $S$ is monotone then $S \subset M_{\varphi s}$ and (in this case is not sure that $\left.c \leq \varphi_{S}\right) S \subset T_{\varphi_{S}}$. Therefore equality holds if $S$ belongs to the class $\mathfrak{M}(X)$ of maximal monotone subsets of $X \times X^{*}$.

On the other hand, simplifying a pioneering approach of Simons ([18]), Penot $([15,16])$ pointed out that one can associate to any subset $S$ of $X \times X^{*}$ the functions $c_{S}:=c+\iota_{s}$, where $\iota_{S}$ is the indicator function of $S$ given by $\iota_{S}(z)=0$ if $z \in S,+\infty$ else, and its convexified functions conv $c_{S}$ and $\psi_{S}:=\overline{\operatorname{conv}} c_{S}=\left(c_{S}\right)^{* *}$ (denoted by $p_{S}$ 
in [16, Proposition 4]). Then $S$ is monotone if and only if conv $c_{S} \geq c$ or, equivalently, conv $c_{s} \in \mathscr{F}$ (see [16, Proposition 4], [23, Proposition 1]); of course, in such a case, $S \subset M_{\text {conv } c_{s}}$. Note that $\left(c_{S}^{*}\right)^{\top}$ is the Fitzpatrick function $\varphi_{S}$ associated to $S$. Moreover, by [16, Proposition 4], a nonempty subset $S \subset X \times X^{*}$ is monotone if and only if there exists $f \in \mathscr{F}$ with $S \subset M_{f}$ if and only if there exists $f \in \mathscr{G}$ with $S \subset M_{f}$.

The following result is practically contained in [5, Theorem 3.1] and [16, Theorem 6]. We give here another proof which does not need the renorming of $X$. It uses the function $\delta$ of the "perfect square trick" of [18, Section 7] given by

$$
\delta\left(x, x^{*}\right):=\frac{1}{2}\|x\|^{2}+\left\langle x, x^{*}\right\rangle+\frac{1}{2}\left\|x^{*}\right\|^{2}, \quad\left(x, x^{*}\right) \in X \times X^{*} .
$$

This function, which is clearly nonnegative, plays an important role in [18, Section 7] and [20].

PROPOSITION 2.1. For any $f \in \mathscr{G}$, the operator $M_{f}$ is maximal monotone if and only if $f$ belongs to $\mathscr{H}$ :

$$
M_{f} \in \mathfrak{M}(X) \quad \Longleftrightarrow f^{*} \geq c^{\top} .
$$

Proof. First observe that for $M:=M_{f}$, we have $f \leq c_{M}$ by the very definitions. It follows that $f^{*} \geq c_{M}^{*}$. Assume now that $M \in \mathfrak{M}(X)$. By [16, Theorem 5] we have that $c_{M}^{*} \geq c^{\top}$, and so $f^{*} \geq c^{\top}$. Conversely, assume that $f^{*} \geq c^{\top}$. Consider $\left(u, u^{*}\right) \in X \times X^{*}$ such that $M \cup\left\{\left(u, u^{*}\right)\right\}$ is monotone, that is, $\left\langle u-x, u^{*}-x^{*}\right\rangle \geq 0$ for every $\left(x, x^{*}\right) \in M$. Taking $g:=f^{* \top}$, since $\delta \geq 0$, we have that $g\left(x, x^{*}\right)-\left\langle x, x^{*}\right\rangle+$ $\delta\left(u-x, u^{*}-x^{*}\right) \geq 0$ for every $\left(x, x^{*}\right) \in X \times X^{*}$. By [20, Lemma 1.3] we get some $\left(x, x^{*}\right) \in X \times X^{*}$ such that $g^{*}\left(x^{*}, x\right)-\left\langle x, x^{*}\right)+\delta\left(u-x, u^{*}-x^{*}\right) \leq 0$. Since $g^{*}\left(x^{*}, x\right)=f\left(x, x^{*}\right)$, it follows that $f\left(x, x^{*}\right) \leq\left\langle x, x^{*}\right\rangle$, hence $\left(x, x^{*}\right) \in M$ and

$$
\delta\left(u-x, u^{*}-x^{*}\right)=\frac{1}{2}\|u-x\|^{2}+\left\langle u-x, u^{*}-x^{*}\right\rangle+\frac{1}{2}\left\|u^{*}-x^{*}\right\|^{2}=0 .
$$

Because $\left\langle u-x, u^{*}-x^{*}\right\rangle \geq 0$ by the choice of $\left(u, u^{*}\right)$, we obtain that $\left(u, u^{*}\right)=$ $\left(x, x^{*}\right) \in M$. Hence $M \in \mathfrak{M}(X)$.

Using Proposition 2.1 we can give an answer to [6, Problem 5.3] which is formulated in the following way: For which convex functions $f$ is $T_{f}$ maximal monotone? Our answer is given in the next corollary.

COROLLARY 2.2. For $f \in \Gamma\left(X \times X^{*}\right)$ the following assertions are equivalent:

(i) $T_{f} \in \mathfrak{M}(X)$;

(ii) $\boldsymbol{g}_{f}^{*} \geq c^{\top}$;

(iii) $f\left(x, x^{*}\right)+f^{*}\left(y^{*}, y\right) \geq \frac{1}{2}\left\langle x+y, x^{*}+y^{*}\right\rangle$ for every $x, y \in X, x^{*}, y^{*} \in X^{*}$. 
ProOF. The equivalence (i) $\Leftrightarrow$ (ii) is a consequence of the preceding proposition and of relation (2.2). The equivalence (ii) $\Leftrightarrow$ (iii) follows from the fact that $g_{f}=\frac{1}{2} f+\frac{1}{2} f^{* \top}$, and so $g_{f}^{*}=\overline{\left(\frac{1}{2} f\right)^{*} \square\left(\frac{1}{2} f^{*}\right)^{*}}$, where $\square$ denotes the infimal convolution operation. Because $c$ is norm-continuous, $g_{f}^{*} \geq c^{\top}$ if and only if $\left(\frac{1}{2} f\right)^{*} \square\left(\frac{1}{2} f^{* \top}\right)^{*} \geq c^{\top}$, that is, (iii) holds, taking into account the definition of $\square$ and the relation $(\lambda h)^{*}\left(z^{*}\right)=\lambda h^{*}\left(\lambda^{-1} z^{*}\right)$ for any function $h, \lambda>0, z^{*}$.

We complete Proposition 2.1 by the next result.

PROPOSITION 2.3. Let $M \subset X \times X^{*}$ be nonempty. Then $M \in \mathfrak{M}(X)$ if and only if there exists $f \in \mathscr{H}$ such that $M=M_{f}$.

Proof. The sufficiency is given by Proposition 2.1. For the necessity, take $M \epsilon$ $\mathfrak{M}(X)$; then, by [16, Theorem 5], we can take $f:=\psi_{M}=\overline{\operatorname{conv}} c_{M}$ or $f:=\varphi_{M}=$ $\left(c_{M}^{*}\right)^{\top}$.

The property of $\psi_{M}$ and $\varphi_{M}$ mentioned in the proof of Proposition 2.3 when $M \in \mathfrak{M}(X)$ is shared by any function in $\mathscr{H}$.

Proposition 2.4. Let $f \in \mathscr{H}$ and let $g:=f^{* \top}$. Then $T_{f}=T_{g}=M_{f}=M_{g}$ and $T_{f} \in \mathfrak{M}(X)$.

Proof. By [6, Theorem 2.4] (recalled above) we have $M_{f} \subset T_{f}$. Let $\left(x, x^{*}\right) \in T_{f}$, that is, $f\left(x, x^{*}\right)+f^{*}\left(x^{*}, x\right)=2\left\langle x, x^{*}\right\rangle$. Since $f \geq c$ and $f^{*} \geq c^{\top}$, we obtain that $f^{* \top}\left(x, x^{*}\right)=f^{*}\left(x^{*}, x\right)=\left\langle x, x^{*}\right)$ and so $\left(x, x^{*}\right) \in M_{g}$. Hence $M_{f} \subset T_{f} \subset$ $M_{g}$. Since $g \in \mathscr{H}$, we can substitute $g$ for $f$ and get $M_{g} \subset T_{g} \subset M_{g^{*}}$. As $g^{* \top}=f$, the announced equalities follow. The fact that $M_{f} \in \mathfrak{M}(X)$ is given in Proposition 2.3.

COROLlaRY 2.5. Let $f \in \mathscr{H}$. Then $g_{f}$ given by (2.3) is also in $\mathscr{H}$ and $M_{f}=M_{g}$.

Proof. By (2.2) and Proposition 2.4 we have that $M:=T_{f}=M_{g f}$ is maximal monotone. Using Proposition 2.1 we obtain that $g_{f}^{*} \geq c^{\top}$, that is, $g_{f} \in \mathscr{H}$.

Having $M \in \mathfrak{M}(X)$, it is natural to ask which are those functions $f \in \mathscr{H}$ with the property that $M=M_{f}$; such a function will be called a representative of $M$. The next result gives the answer.

PROPOSITION 2.6. Let $M \in \mathfrak{M}(X)$ and $f \in \mathscr{H}$. Then $M=M_{f}$ if and only if $\varphi_{M} \leq f \leq \psi_{M}$. 
PROOF. If $\varphi_{M} \leq f \leq \psi_{M}$, as $M_{\varphi_{M}}=M_{\psi_{M}}=M$ by [16, Theorem 5], we obtain that $M_{f}=M$. Conversely, assume that $M=M_{f}$. Then we have that $c_{M} \geq f$ and so $f \leq \overline{\operatorname{conv}} c_{M}=\psi_{M}$. By Proposition 2.4 we have also that $M=M_{f} \cdot$ and so $f^{* \top} \leq \psi_{M}$. By conjugation we get $f \geq\left(\psi_{M}^{\top}\right)^{*}=\varphi_{M}$.

Fitzpatrick's Problem 5.1 in [6] is formulated as follows: For which convex functions $f$ is $\varphi_{T_{f}}=f$ ?

If $\varphi_{T_{f}}=f$, then $f$ is necessarily lsc; moreover, because for a monotone multifunction $M$ we have that $\varphi_{M} \leq \psi_{M}=\left(\varphi_{M}\right)^{*}$ [16, Proposition 4], we must have that $f \leq f^{* \top}$. Hence, if $\varphi_{T_{f}}=f$ then $f=\overline{\text { conv }} f \leq f^{* \top}$. However, this condition is not sufficient: just take $f\left(x, x^{*}\right):=\|x\|^{2} / 2+\left\|x^{*}\right\|^{2} / 2$ on a Hilbert space $X$; then $f^{* \top}=f \geq c, g_{f}=f$ and thus $T_{f}=M_{g_{f}}=M_{f}=\Delta_{x}:=\{(x, x) \mid x \in X\}$. However $\varphi_{\Delta_{x}}\left(x, x^{*}\right)=\left\|x+x^{*}\right\|^{2} / 4$ by [16, Example 2]. But we can give a complete answer for functions $f \in \mathscr{H}$; in fact, part of it already appears in [6, Theorem 3.7] as a necessary condition.

COROLlary 2.7. Let $f, g \in \mathscr{H}$. Then $\varphi_{T_{f}}=f$ (respectively $\psi_{T_{s}}=g$ ) if and only if $f$ is minimal (respectively $g$ is maximal) in $\mathscr{H}$ with respect to $\leq$.

Proof. Assume first that $\varphi_{T_{f}}=f$ and take $g \in \mathscr{H}$ such that $g \leq f$. It follows that $M_{f} \subset M_{g}$. By Proposition 2.3, $M_{f}$ and $M_{g}$ are maximal monotone and so $M_{f}=M_{g}$. By Proposition 2.4, $M:=T_{f}=M_{f}=M_{g}$. Then, by Proposition 2.6 we obtain that $f=\varphi_{M} \leq g \leq \psi_{M}$. Hence $f$ is minimal.

Assume now that $f$ is minimal in $\mathscr{H}$. By Proposition 2.3 we have that $M:=$ $M_{f}$ is maximal monotone, from Proposition 2.4 we have that $M=T_{f}$, and from Proposition 2.6 we have that $\varphi_{M} \leq f$. Since $\varphi_{M} \in \mathscr{H}$ and $f$ is minimal, it follows that $f=\varphi_{M}=\varphi_{T_{j}}$.

The assertion concerning $g$ follows from what precedes by setting $f=g^{* \top}$ and using the fact that $T_{f}=T_{g}$ by (2.1) and that $\varphi_{M}^{* \top}=\psi_{M}$ for every $M \subset X \times X^{*}$.

Note that $[f \in \mathscr{H}, g \in \mathscr{G}, g \leq f] \Rightarrow g \in \mathscr{H}$. This is because $g^{*} \geq f^{*} \geq c$. Thus, if $f \in \mathscr{G}$ is such that $\varphi_{T_{f}}=f$ then $f$ is minimal in $\mathscr{G}$ with respect to $\leq$. Indeed, as observed above we have $f \leq f^{*}$, and so $f \in \mathscr{H}$; the conclusion follows from Corollary 2.7.

Let us subsume the preceding analysis in the following statement.

COROLlaRY 2.8. The mappings $\varphi: M \mapsto \varphi_{M}$ and $\psi: M \mapsto \psi_{M}$ define bijections from the set $\mathfrak{M}(X)$ of maximal monotone operators on $X$ onto the sets $\mathscr{H}_{m}$ and $\mathscr{H}^{m}$ of minimal and maximal elements of $\mathscr{H}$, respectively, with inverses $T: f \mapsto T_{f}=M_{f}$. Moreover $\varphi$ is antitone (that is, reverses order) and $\psi$ is homotone (that is, preserves order). For each $M \in \mathfrak{M}(X)$, the inverse image $T^{-1}(M)$ of $T: \mathscr{H} \rightarrow \mathfrak{M}(X)$ is the segment $\left[\varphi_{M}, \psi_{M}\right]$. 
Burachik and Svaiter $[4,5]$ considered, for $M \in \mathfrak{M}(X)$, the class

$$
\mathscr{H}(M):=\left\{f \in \mathscr{G} \mid M \subset M_{f}\right\} .
$$

In [6, Theorem 3.10] it is proved that $\varphi_{M}$ is minimal in the class $\mathscr{H}(M)$, a property weaker than Corollary 2.7. Propositions 2.3 and 2.6 show that for $M \in \mathfrak{M}(X)$,

$$
\mathscr{H}(M)=\left\{f \in \mathscr{H} \mid M=M_{f}\right\}=\left\{f \in \Gamma\left(X \times X^{*}\right) \mid \varphi_{M} \leq f \leq \psi_{M}\right\} .
$$

The first equality is the main result of [5]; since $M_{f}$ is monotone for each $f \in \mathscr{F}$, as recalled above, given $f \in \mathscr{H}(M)$, we have $M=M_{f}$ by maximality. The fact that $\varphi_{M} \in \mathscr{H}(M)$ and $\varphi_{M} \leq f$ for every $f \in \mathscr{H}(M)$ was obtained in [6, Theorem 3.10] (see also [5, Theorem 1.1]); the inequality $f \leq \psi_{M}$ can easily be deduced from [6, Proposition 4.2].

From the preceding equality, we obtain also that $f \in \mathscr{H}(M) \Rightarrow f^{* \top} \in \mathscr{H}(M)$, a result established in [4]. By Proposition 2.6, the function $\sigma_{T}$ defined in the beginning of $\left[21\right.$, Section 2] coincides with $\psi_{M}$ when $X$ is reflexive.

Martínez-Legaz and Théra [12] introduced the class of functions

$$
\Phi(X):=\left\{f \in \Gamma\left(X \times X^{*}\right) \mid\left(f+\iota_{B(f)}\right)^{*}=f^{\top}\right\},
$$

where

$$
B(f):=\left\{\left(x, x^{*}\right) \in X \times X^{*} \mid f\left(x, x^{*}\right) \leq\left\langle x, x^{*}\right)\right\} .
$$

Note that for $f \in \Phi(X)$ (see also [12, Proposition 1])

$$
B(f) \neq \emptyset, \quad f \geq c, \quad f+\iota_{B(f)}=c_{B(f)}, \quad f^{*} \geq c^{\top} .
$$

Indeed, if $B(f)=\emptyset$ then $f+\iota_{B(f)} \equiv \infty$, and so $f \equiv-\infty$. To prove that $f \geq c$, we consider two cases. If $\left(x, x^{*}\right) \notin B(f)$ we have that $f\left(x, x^{*}\right)>\left\langle x, x^{*}\right\rangle=c\left(x, x^{*}\right)$. Let $\left(x, x^{*}\right) \in B(f)$. Then

$$
\begin{aligned}
2 f\left(x, x^{*}\right) & =\left(f+\iota_{B(f)}\right)\left(x, x^{*}\right)+\left(f+\iota_{B(f)}\right)^{*}\left(x^{*}, x\right) \\
& \geq\left\langle\left(x, x^{*}\right),\left(x^{*}, x\right)\right\rangle=2\left(x, x^{*}\right) .
\end{aligned}
$$

Hence $f\left(x, x^{*}\right) \geq\left\langle x, x^{*}\right\rangle$. Since $f \geq c$ we have $f+\iota_{B(f)}=c_{B(f)}$ and conversely this equality yields $f \geq c$. Because $f \leq f+\iota_{B(f)}$, we have that

$$
f^{*} \geq\left(f+\iota_{B(f)}\right)^{*}=f^{\top} \geq c^{\top} .
$$

It follows that $\Phi(X) \subset \mathscr{H}$ and $B(f)=M_{f}$. Moreover if $f \in \Phi(X)$ then, by Proposition 2.3, we have that $M:=M_{f}$ is maximal monotone and $M=B(f)$ since $f \geq c$; as $f+\iota_{M}=c_{M}$, by definition of $M_{f}$, we get $f^{\top}=c_{M}^{*}$, that is, $f=\varphi_{M}$. Hence 
$\Phi(X) \subset\left\{\varphi_{M} \mid M \in \mathfrak{M}(X)\right\}$. Because for $M \in \mathfrak{M}(X)$ we have that $\varphi_{M} \in \Gamma\left(X \times X^{*}\right)$ and $M_{\varphi_{M}}=M$ as recalled above, we obtain that $\left(\varphi_{M}+\iota_{B\left(\varphi_{M}\right)}\right)^{*}=c_{M}^{*}=\varphi_{M}^{\top}$, and so

$$
\Phi(X)=\left\{\varphi_{M} \mid M \in \mathfrak{M}(X)\right\} .
$$

This is practically [12, Theorem 2] which is stated for $X$ a Banach space; one must observe that in this case, in the definition of $\Phi(X)$ one must endow $X^{*}$ with the weak ${ }^{*}$ topology (and the result remains valid for $X$ a separated locally convex space).

Now let us introduce the class of functions

$$
\Psi(X):=\left\{g \in \Gamma\left(X \times X^{*}\right) \mid\left(g^{*}+\iota_{B\left(g^{*}\right)}\right)^{* *}=g\right\},
$$

where, as above,

$$
B\left(g^{*}\right):=\left\{\left(x, x^{*}\right) \in X \times X^{*} \mid g^{*}\left(x^{*}, x\right) \leq\left\langle x, x^{*}\right\rangle\right\} .
$$

Note that for $g \in \Psi(X)$ the function $f:=g^{* \top}$ belongs to $\Phi(X)$. Conversely, for any $f \in \Phi(X)$ we have $f^{* \top} \in \Psi(X)$. It follows that

$$
\Psi(X)=\left\{\psi_{M} \mid M \in \mathfrak{M}(X)\right\} .
$$

Thus, for each $g \in \Psi(X)$ we have

$$
B(g) \neq \emptyset, \quad g \geq c, \quad g+\iota_{B(g)}=c_{B(g)}, \quad g^{*} \geq c^{\top} .
$$

\section{Questions about operations}

Let $M, N: X \rightrightarrows X^{*}$ be monotone multifunctions and $\alpha>0$. Then the multifunctions $\alpha M$ and $M+N$ are defined by $(\alpha M)(x):=\alpha \cdot M(x)$ and $(M+N)(x):=$ $M(x)+N(x)$. Of course, $\operatorname{dom}(\alpha M)=\operatorname{dom} M, \operatorname{dom}(M+N)=\operatorname{dom} M \cap \operatorname{dom} N$, and $\alpha M, M+N$ are monotone; $\alpha M$ is maximal monotone when $M$ is so, but $M+N$ is not necessarily maximal monotone when $M$ and $N$ are so. For every $\left(x, x^{*}\right) \in X \times X^{*}$ we have

$$
\begin{aligned}
\varphi_{\alpha M}\left(x, x^{*}\right) & =\sup \left\{\left\langle x, u^{*}\right\rangle+\left\langle u, x^{*}\right\rangle-\left\langle u, u^{*}\right\rangle \mid\left(u, u^{*}\right) \in \operatorname{gph}(\alpha M)\right\} \\
& =\sup \left\{\left(x, \alpha w^{*}\right\rangle+\left\langle w, x^{*}\right\rangle-\left\langle w, \alpha w^{*}\right\rangle \mid\left(w, w^{*}\right) \in \operatorname{gph} M\right\} \\
& =\alpha \varphi_{M}\left(x, \alpha^{-1} x^{*}\right) .
\end{aligned}
$$

For what concerns the sum, we need to use a partial convolution for functions on a product space, as in [16] and [20]. Consider $g, h: X \times Y \rightarrow \overline{\mathbb{R}}$ and define $g \square_{2} h: X \times Y \rightarrow \overline{\mathbb{R}}$ by

$$
\left(g \square_{2} h\right)(x, y):=\inf \left\{g\left(x, y_{1}\right)+h\left(x, y_{2}\right) \mid y_{1}, y_{2} \in Y, y=y_{1}+y_{2}\right\} ;
$$


$g \square_{1} h$ is defined similarly. Then

$$
\begin{aligned}
\left(g \square_{2} h\right)^{*}\left(x^{*}, y^{*}\right)= & \sup \left\{\left(x, x^{*}\right\rangle+\left\langle y, y^{*}\right\rangle\right. \\
& \left.-\inf \left\{g\left(x, y_{1}\right)+h\left(x, y_{2}\right) \mid y=y_{1}+y_{2}\right\} \mid(x, y) \in X \times Y\right\} \\
= & \sup \left\{\left\langle x, x^{*}\right\rangle+\left\langle y_{1}+y_{2}, y^{*}\right\rangle\right. \\
& \left.-g\left(x, y_{1}\right)-h\left(x, y_{2}\right) \mid x \in X, y_{1}, y_{2} \in Y\right\} \\
\leq & \sup \left\{\left\langle x, x_{1}^{*}\right\rangle+\left\langle y_{1}, y^{*}\right\rangle-g\left(x, y_{1}\right) \mid x \in X, y_{1} \in Y\right\} \\
& +\sup \left\{\left(x, x_{2}^{*}\right\rangle+\left\langle y_{2}, y^{*}\right\rangle-h\left(x, y_{2}\right) \mid x \in X, y_{2} \in Y\right\}
\end{aligned}
$$

for all $x_{1}^{*}, x_{2}^{*} \in X^{*}$ with $x_{1}^{*}+x_{2}^{*}=x^{*}$. Hence

$$
\left(g \square_{2} h\right)^{*}\left(x^{*}, y^{*}\right) \leq\left(g^{*} \square_{1} h^{*}\right)\left(x^{*}, y^{*}\right) \quad \forall\left(x^{*}, y^{*}\right) \in X^{*} \times Y^{*} .
$$

It would be interesting to know when $\left(g \square_{2} h\right)^{*}=\overline{g^{*} \square_{1} h^{*}}$. In [20, Theorem 4.2] it is shown that equality holds in (3.1) and the infimum is attained in the definition of $g^{*} \square_{1} h^{*}$ when $X, Y$ are Banach spaces, $g, h \in \Gamma(X \times Y)$ and $0 \in{ }^{i c}\left(\operatorname{Pr}_{X}(\operatorname{dom} g)-\right.$ $\operatorname{Pr}_{X}(\operatorname{dom} h)$ ); hereafter, as in [22, page 15], for a subset $S$ of $X$ we write $x \in{ }^{i c} S$ to mean that the affine manifold $A:=$ aff $S$ generated by $S$ is closed and $x$ belongs to the relative algebraic interior ${ }^{i} S$ of $S$ (that is the algebraic interior of $S$ in $A: x \in{ }^{i} S$ if and only if for every $a \in A$ there exists some $\varepsilon>0$ such that $x+t(a-x) \in S$ for $t \in[-\varepsilon, \varepsilon])$. Thus ${ }^{i c} S$ is empty when aff $S$ is not closed.

As observed in [16], from the very definitions, we have that $c_{M+N}=c_{M} \square_{2} c_{N}$, and so, by (3.1), and the fact that $\psi_{M} \square_{2} \psi_{N}$ is convex and less than $c_{M+N}$,

$$
\varphi_{M+N} \leq \overline{\varphi_{M} \square_{2} \varphi_{N}}, \quad \psi_{M+N} \geq \overline{\psi_{M} \square_{2} \psi_{N}} .
$$

In the next result we adapt the proof of [20, Lemma 5.3 (b)] to the case of a maximal monotone multifunction defined on $X \times Y$, where $Y$ is another reflexive Banach space.

LEMMA 3.1. Let $F \in \mathfrak{M}(X \times Y)$, let $Y_{0} \subset Y$ be a closed linear subspace and $y_{0} \in Y$ be such that $\operatorname{Pr}_{Y}(F) \subset y_{0}+Y_{0}$. Then $\operatorname{Pr}_{Y}\left(\operatorname{dom} \varphi_{F}\right) \subset y_{0}+Y_{0}$.

PROOF. Let $y \in \operatorname{Pr}_{Y}\left(\operatorname{dom} \varphi_{F}\right)$; then there exist $x \in X, x^{*} \in X^{*}, y^{*} \in Y^{*}$ and $\gamma \in \mathbb{R}$ such that $\left(x, y, x^{*}, y^{*}, \gamma\right) \in \operatorname{epi} \varphi_{F}$. Fix $\left(u, v, u^{*}, v^{*}\right) \in F$ and take an arbitrary $\left(u^{\prime}, v^{\prime}, u^{* \prime}, v^{* \prime}\right) \in F$. Then, for every $z^{*} \in Y_{0}^{\perp}$ one has $\left(v-v^{\prime}, z^{*}\right)=0$ and

$$
\begin{aligned}
& \left\langle(u, v)-\left(u^{\prime}, v^{\prime}\right),\left(u^{*}, v^{*}+z^{*}\right)-\left(u^{* \prime}, v^{* \prime}\right)\right\rangle \\
& =\left\langle(u, v)-\left(u^{\prime}, v^{\prime}\right),\left(u^{*}, v^{*}\right)-\left(u^{* \prime}, v^{* \prime}\right)\right\rangle+\left\langle v-v^{\prime}, z^{*}\right\rangle \geq 0 .
\end{aligned}
$$

Since $F$ is maximal monotone, we obtain that $\left(u, v, u^{*}, v^{*}+z^{*}\right) \in F$. Hence the definition of $\varphi_{F}$ as $c_{F}^{*}$ and the choice of $x, x^{*}, y^{*}, \gamma$ show that

$$
\gamma \geq\left\langle(x, y),\left(u^{*}, v^{*}+z^{*}\right)\right\rangle+\left\langle(u, v),\left(x^{*}, y^{*}\right)\right\rangle-\left\langle(u, v),\left(u^{*}, v^{*}+z^{*}\right)\right\rangle
$$


for every $z^{*} \in Y_{0}^{\perp}$, which implies that $\left\langle y-v, z^{*}\right\rangle=0$ for each $z^{*} \in Y_{0}^{\perp}$. It follows that $y-v \in\left(Y_{0}^{\perp}\right)^{\perp}=Y_{0}$. Hence $y \in v+Y_{0} \subset \operatorname{Pr}_{Y}(F)+Y_{0} \subset y_{0}+Y_{0}+Y_{0}=y_{0}+Y_{0}$.

The next result is an easy consequence of [22, Theorem 2.8.6].

LEMMA 3.2. Let $f \in \Lambda\left(X \times Y \times X^{*} \times Y^{*}\right)$ and let $g: X \times X^{*} \rightarrow \mathbb{\mathbb { R }}$ be given by

$$
g\left(x, x^{*}\right):=\inf \left\{f\left(x, 0, x^{*}, y^{*}\right) \mid y^{*} \in Y^{*}\right\} .
$$

Then $g$ is convex; moreover, if $f$ is $l s c$ and $0 \in{ }^{i c}\left(\operatorname{Pr}_{Y}(\operatorname{dom} f)\right)$ then

$$
g^{*}\left(u^{*}, u\right)=\min \left\{f^{*}\left(u^{*}, v^{*}, u, 0\right) \mid v^{*} \in Y^{*}\right\} \quad \forall u \in X, \forall u^{*} \in X^{*} .
$$

PROOF. It is obvious that $g$ is convex (as the performance function associated with a convex function).

Assume that $f$ is lsc and $0 \in{ }^{i c}\left(\operatorname{Pr}_{Y}(\operatorname{dom} f)\right)$. Consider

$$
\mathscr{C}: X \times X^{*} \rightrightarrows X \times Y \times X^{*} \times Y^{*}, \mathscr{C}\left(x, x^{*}\right):=\{x\} \times\{0\} \times\left\{x^{*}\right\} \times Y^{*} .
$$

Then $\mathscr{C}$ is a closed convex process (in fact its graph is a closed linear subspace) and $g\left(x, x^{*}\right)=\inf \left\{f\left(u, v, u^{*}, v^{*}\right) \mid\left(u, v, u^{*}, v^{*}\right) \in \mathscr{C}\left(x, x^{*}\right)\right\}$. Moreover,

$$
\operatorname{dom} f-\operatorname{Im} \mathscr{C}=X \times \operatorname{Pr}_{Y}(F) \times X^{*} \times Y^{*},
$$

which shows that $0 \in{ }^{i c}(\operatorname{dom} f-\operatorname{Im} \mathscr{C})$. Applying [22, Theorem 2.8.6(v)] we obtain that $g^{*}\left(u^{*}, u\right)=\min \left\{f^{*}\left(x^{*}, y^{*}, x, y\right) \mid\left(u^{*}, u\right) \in \mathscr{C}^{*}\left(x^{*}, y^{*}, x, y\right)\right\}$. But a simple calculation shows that $\mathscr{C}^{*}\left(x^{*}, y^{*}, x, y\right)=\left\{\left(x^{*}, x\right)\right\}$ if $y=0$ and $\mathscr{C}^{*}\left(x^{*}, y^{*}, x, y\right)=\emptyset$ else. The conclusion is now obvious.

ANOTHER PROOF. Observe that $-g^{*}\left(u^{*}, u\right)$ is equal to

$$
\inf \left\{f\left(x, y, x^{*}, y^{*}\right)-\left\langle u, x^{*}\right\rangle-\left\langle x, u^{*}\right\rangle \mid y=0, x \in X, x^{*} \in X^{*}, y^{*} \in Y^{*}\right\}
$$

so that, using the Lagrange multiplier rule which is satisfied under the assumption $0 \in{ }^{i c}\left(\operatorname{Pr}_{Y}(\operatorname{dom} f)\right)$ in order to get rid of the linear constraint $\operatorname{Pr}_{Y}\left(x, y, x^{*}, y^{*}\right)=0$, there exists some multiplier $\bar{v}^{*} \in Y^{*}$ such that

$$
\begin{aligned}
-g^{*}\left(u^{*}, u\right)= & \inf \left\{f\left(x, y, x^{*}, y^{*}\right)-\left\langle u, x^{*}\right\rangle-\left\langle x, u^{*}\right\rangle-\left\langle y, \bar{v}^{*}\right\rangle \mid\right. \\
& \left.x \in X, y \in Y, x^{*} \in X^{*}, y^{*} \in Y^{*}\right\} \\
= & \max _{v^{*} \in Y^{*}} \inf \left\{f\left(x, y, x^{*}, y^{*}\right)-\left\langle u, x^{*}\right\rangle-\left\langle x, u^{*}\right\rangle-\left\langle y, v^{*}\right\rangle \mid\right. \\
& \left.x \in X, y \in Y, x^{*} \in X^{*}, y^{*} \in Y^{*}\right\} \\
= & -\min \left\{f^{*}\left(u^{*}, v^{*}, u, 0\right) \mid v^{*} \in Y^{*}\right\} .
\end{aligned}
$$


The next result corresponds to [17, Theorem 4.1] and [20, Lemma 5.1]; we use the notation ri $S$ for the topological interior of $S$ w.r.t. $A:=\operatorname{cl}($ aff $S$ ) (so ri $S$ is empty if aff $S$ is not closed). Thus one always has ri $S \subset{ }^{i c} S$. In the proof, we use the facts that when $C$ is convex and ${ }^{i c} C$ is nonempty, we have aff $C=$ aff ${ }^{i c} C$ and moreover,

$$
{ }^{i c} C \subset S \subset C \Longrightarrow{ }^{i c} C={ }^{i c} S \text {. }
$$

Lemma 3.3. Let $F \subset X \times Y \times X^{*} \times Y^{*}$ be monotone. Then

$$
G:=\left\{\left(x, x^{*}\right) \in X \times X^{*} \mid \exists y^{*} \in Y^{*}:\left(x, 0, x^{*}, y^{*}\right) \in F\right\}
$$

is monotone, too. Moreover, if $F$ is maximal monotone, then

$$
{ }^{i c}\left(\operatorname{Pr}_{Y}\left(\operatorname{dom} \varphi_{F}\right)\right)={ }^{i c}\left(\operatorname{Pr}_{Y}(F)\right)=\operatorname{ri}\left(\operatorname{Pr}_{Y}(F)\right) .
$$

Furthermore, if $0 \in{ }^{i c}\left(\operatorname{Pr}_{Y}(F)\right)$ then $G$ is maximal monotone.

PROOF. For showing the monotonicity of $G$ consider $\left(x, x^{*}\right),\left(u, u^{*}\right) \in G$; then there exist $y^{*}, v^{*} \in Y^{*}$ such that $\left(x, 0, x^{*}, y^{*}\right),\left(u, 0, u^{*}, v^{*}\right) \in F$. We obtain that

$$
0 \leq\left\langle(x, 0)-(u, 0),\left(x^{*}, y^{*}\right)-\left(u^{*}, v^{*}\right)\right\rangle=\left\langle x-u, x^{*}-u^{*}\right\rangle .
$$

Let $F$ be maximal monotone. Assume first that $0 \in{ }^{i r}\left(\operatorname{Pr}_{Y}\left(\operatorname{dom} \varphi_{F}\right)\right)$ and consider the function

$$
\zeta: X \times X^{*} \rightarrow \overline{\mathbb{B}}, \quad \zeta\left(x, x^{*}\right):=\inf \left\{\varphi_{F}\left(x, 0, x^{*}, y^{*}\right) \mid y^{*} \in Y^{*}\right\}
$$

Then, by Lemma 3.2, we obtain that

$$
\zeta^{*}\left(u^{*}, u\right)=\min \left\{\varphi_{F}^{*}\left(u^{*}, v^{*}, u, 0\right) \mid v^{*} \in Y^{*}\right\} \quad \forall u \in X, u^{*} \in X^{*} .
$$

Since $\varphi_{F} \geq c, \varphi_{F}^{*} \geq c^{\top}$, one has $\varphi_{F}\left(x, 0, x^{*}, y^{*}\right) \geq\left\langle(x, 0),\left(x^{*}, y^{*}\right)\right\rangle=\left\langle x, x^{*}\right\rangle$, whence $\zeta\left(x, x^{*}\right) \geq\left\langle x, x^{*}\right\rangle$, and $\varphi_{F}^{*}\left(u^{*}, v^{*}, u, 0\right) \geq\left\langle(u, 0),\left(u^{*}, v^{*}\right)\right\rangle=\left\langle u, u^{*}\right\rangle$, whence $\zeta^{*}\left(u^{*}, u\right) \geq\left\langle u, u^{*}\right\rangle$. Hence $\zeta^{* \top} \in \mathscr{H}$. In order to have that $G \in \mathfrak{M}(X)$, by Proposition 2.3, it is sufficient to show that $G=M_{\xi^{*}}$. If $\left(x, x^{*}\right) \in G$ then $\left(x, 0, x^{*}, y^{*}\right) \in F$ for some $y^{*} \in Y^{*}$. Hence, by Proposition 2.3 with $f=\psi_{F}=\varphi_{F}^{*}$,

$$
\zeta^{* \top}\left(x, x^{*}\right) \leq \varphi_{F}^{*}\left(x^{*}, y^{*}, x, 0\right)=\left\langle(x, 0),\left(x^{*}, y^{*}\right)\right\rangle=\left\langle x, x^{*}\right\rangle,
$$

which implies that $\left(x, x^{*}\right) \in M_{\zeta^{* \top}}$. Conversely, if $\left(x, x^{*}\right) \in M_{\zeta^{* \top}}$ then $\zeta^{* \top}\left(x, x^{*}\right)=$ $\left\langle x, x^{*}\right\rangle$. From (3.7) we get some $y^{*} \in Y^{*}$ with $\varphi_{F}^{*}\left(x^{*}, y^{*}, x, 0\right)=\left\langle x, x^{*}\right\rangle=$ $\left((x, 0),\left(x^{*}, y^{*}\right)\right)$. Again using Proposition 2.3 with $f=\psi_{F}=\varphi_{F}^{*}$, we obtain that $\left(x, 0, x^{*}, y^{*}\right) \in F$, and so $\left(x, x^{*}\right) \in G$. Hence $G$ is maximal monotone. In particular, $G$ is nonempty, and so $0 \in \operatorname{Pr}_{Y}(F)$. 
Let $y \in{ }^{i c}\left(\operatorname{Pr}_{Y}\left(\operatorname{dom} \varphi_{F}\right)\right)$. Replacing $F$ by $F^{\prime}:=F-(0, y, 0,0)$, it is obvious that $G$ is replaced by $G^{\prime}=\left\{\left(x, x^{*}\right) \in X \times X^{*} \mid \exists y^{*} \in Y^{*}:\left(x, y, x^{*}, y^{*}\right) \in F\right\}$, and, by [20, Lemma 5.4 (b)], $0 \in{ }^{i c}\left(\operatorname{Pr}_{Y}\left(\operatorname{dom} \varphi_{F^{\prime}}\right)\right)$. By what precedes, it follows that $G^{\prime}$ is maximal monotone. In particular $y \in \operatorname{Pr}_{Y}(F)$. Hence ${ }^{i c}\left(\operatorname{Pr}_{Y}\left(\operatorname{dom} \varphi_{F}\right)\right) \subset \operatorname{Pr}_{Y}(F) \subset$ $\operatorname{Pr}_{Y}\left(\operatorname{dom} \varphi_{F}\right)$. Thus, if ${ }^{i c}\left(\operatorname{Pr}_{Y}\left(\operatorname{dom} \varphi_{F}\right)\right)$ is nonempty, by (3.4) the first equality in (3.6) holds.

Assume now that ${ }^{i c}\left(\operatorname{Pr}_{Y}(F)\right)$ is nonempty and take $y \in{ }^{i c}\left(\operatorname{Pr}_{Y}(F)\right)$. Then $Y_{0}:=$ aff $\left(\operatorname{Pr}_{Y}(F)\right)-y$ is a closed linear space. By Lemma 3.1 we obtain that $\operatorname{Pr}_{Y}\left(\operatorname{dom} \varphi_{F}\right) \subset$ $y+Y_{0}$. Since $\operatorname{Pr}_{Y}(F) \subset \operatorname{Pr}_{Y}\left(\operatorname{dom} \varphi_{F}\right)$, we obtain that

$$
\operatorname{aff}\left(\operatorname{Pr}_{Y}\left(\operatorname{dom} \varphi_{F}\right)\right)=y+Y_{0}=\operatorname{aff}\left(\operatorname{Pr}_{Y}(F)\right) .
$$

It follows that ${ }^{i c}\left(\operatorname{Pr}_{Y}(F)\right) \subset{ }^{i c}\left(\operatorname{Pr}_{Y}\left(\operatorname{dom} \varphi_{F}\right)\right)$, and so ${ }^{i c}\left(\operatorname{Pr}_{Y}\left(\operatorname{dom} \varphi_{F}\right)\right) \neq \emptyset$. Thus, the argument of the preceding paragraph shows that the first equality in (3.6) holds.

Since $\varphi_{F}$ is lsc and the spaces are complete, by [22, Proposition 3.1.5] (with $A=0$ ), we have that ${ }^{i c}\left(\operatorname{Pr}_{Y}\left(\operatorname{dom} \varphi_{F}\right)\right)=\operatorname{ri}\left(\operatorname{Pr}_{Y}\left(\operatorname{dom} \varphi_{F}\right)\right)$ and so (3.6) holds.

The preceding proof yields $\zeta^{* \top}$ as a representative function of the new operator $G$. That output and the whole of the preceding lemma can be given with an arbitrary representative $f$ of $F$ instead of $\varphi_{F}$.

PROPOSITION 3.4. Let $F \in \mathfrak{M}(X \times Y)$ and $f \in \Gamma\left(X \times Y \times X^{*} \times Y^{*}\right)$ be such that $\varphi_{F} \leq f \leq \psi_{F}$. Then

$$
\operatorname{ri}\left(\operatorname{Pr}_{Y}(F)\right)={ }^{i c}\left(\operatorname{Pr}_{Y}(F)\right)={ }^{i c}\left(\operatorname{Pr}_{Y}(\operatorname{dom} f)\right) .
$$

Moreover, if $0 \in{ }^{i c}\left(\operatorname{Pr}_{Y}(F)\right)$, or equivalently $0 \in{ }^{i c}\left(\operatorname{Pr}_{Y}(\operatorname{dom} f)\right)$, then $G$ defined by (3.5) is maximal monotone, the function $g: X \times X^{*} \rightarrow \overline{\mathbb{R}}$, given by

$$
g\left(x, x^{*}\right):=\inf \left\{f\left(x, 0, x^{*}, y^{*}\right) \mid y^{*} \in Y^{*}\right\}
$$

is a representative of $G$ (that is, $g \in \mathscr{H}$ and $G=M_{g}$ ) and the infimum in (3.9) is attained.

PROOF. We first prove that (3.8) holds. Because $c_{F} \geq \psi_{F} \geq f \geq \varphi_{F}$, we have that $F \subset \operatorname{dom} \psi_{F} \subset \operatorname{dom} f \subset \operatorname{dom} \varphi_{F}$, and so

$$
\operatorname{Pr}_{Y}(F) \subset \operatorname{Pr}_{Y}\left(\operatorname{dom} \psi_{F}\right) \subset \operatorname{Pr}_{Y}(\operatorname{dom} f) \subset \operatorname{Pr}_{Y}\left(\operatorname{dom} \varphi_{F}\right) .
$$

When ${ }^{i c}\left(\operatorname{Pr}_{Y}(F)\right)$ is nonempty (3.8) is immediate from (3.10) and (3.6).

Assume now that $y \in{ }^{i c}\left(\operatorname{Pr}_{Y}(\operatorname{dom} f)\right)$. Then $A_{0}:=\operatorname{aff} C$, with $C:=\operatorname{Pr}_{Y}(\operatorname{dom} f)$, is a closed affine manifold and the relative algebraic interior ${ }^{i} C$ of $C$ in $A_{0}$ coincides with ${ }^{i c} C$. Again applying Lemma 3.1 (with $Y_{0}:=A_{0}-y$ ) we obtain

$$
\operatorname{Pr}_{Y}\left(\operatorname{dom} \varphi_{F}\right) \subset A_{0}=y+Y_{0} .
$$


From (3.10) we obtain first that $\operatorname{aff}\left(\operatorname{Pr}_{Y}\left(\operatorname{dom} \varphi_{F}\right)\right)=A_{0}$, then that

$$
{ }^{i}\left(\operatorname{Pr}_{Y}(\operatorname{dom} f)\right) \subset \subset^{i}\left(\operatorname{Pr}_{Y}\left(\operatorname{dom} \varphi_{F}\right)\right) \text {. }
$$

Again using (3.6) we have that ${ }^{i c}\left(\operatorname{Pr}_{Y}(F)\right)$ is nonempty, and so (3.8) holds.

Now assume that $0 \in{ }^{i c}\left(\operatorname{Pr}_{Y}(F)\right)$, or equivalently $0 \in{ }^{i c}\left(\operatorname{Pr}_{Y}(\operatorname{dom} f)\right)$. Then Lemma 3.2 ensures that

$$
g^{*}\left(x^{*}, x\right)=\min \left\{f^{*}\left(x^{*}, y^{*}, x, 0\right) \mid y^{*} \in Y^{*}\right\} \quad \forall x \in X, \forall x^{*} \in X^{*} .
$$

But, by Proposition $2.4, f^{* \top}$ is also a representative of $F$, and so, applying the preceding argument with $f$ replaced by $f^{* \top}$, we find that $g^{* *}\left(x, x^{*}\right)=\min \left\{f^{* *}\left(x, 0, x^{*}, y^{*}\right)\right\}$ $\left.y^{*} \in Y^{*}\right\}=g\left(x, x^{*}\right)$ as $f=f^{* *}$ and the infimum in (3.9) is attained. Thus $g \in \Gamma\left(X \times X^{*}\right)$ and, as in the proof of the preceding lemma, we check that $g \geq c$ and $g^{*} \geq c^{\top}$, that is, $g \in \mathscr{H}$ and that $M_{g}=G$.

Now, let us focus our attention on a construction which involves an element $A$ of the space $L(X, Y)$ of continuous linear operators from $X$ into $Y$ and a monotone multifunction $F: X \times Y \rightrightarrows X^{*} \times Y^{*}$. It is inspired by a classical scheme in convex duality. As in [17, Section 5], define $F_{A}: X \times Y \rightrightarrows X^{*} \times Y^{*}$ by

$$
\operatorname{gph} F_{A}:=\left\{\left(x, y, x^{*}, y^{*}\right) \in X \times Y \times X^{*} \times Y^{*} \mid\left(x^{*}-A^{\top} y^{*}, y^{*}\right) \in F(x, A x+y)\right\} .
$$

Thus, setting $B(x, y):=(x, y+A x)$, we have $F_{A}(x, y)=B^{\top} F B(x, y)$ and $F_{A}$ is monotone. Moreover, since $B$ is an isomorphism with inverse given by $B^{-1}(u, v)=$ $(u, v-A u), F_{A}$ is maximal monotone whenever $F$ is maximal monotone. Indeed, if $M: X \times Y \rightrightarrows X^{*} \times Y^{*}$ is a monotone operator whose graph contains the graph of $F_{A}$, then $\left(B^{-1}\right)^{\top} M B^{-1}$ is a monotone operator whose graph contains the graph of $F$. Now, since for an isomorphism $L: U \rightarrow V$ between two Banach spaces one has

$$
\forall\left(u, u^{*}\right) \in U \times U^{*}: c\left(L u,\left(L^{-1}\right)^{\top} u^{*}\right)=c\left(u, u^{*}\right)
$$

and since for $g: V \rightarrow \mathbb{R}$

$$
(g \circ L)^{*}=g^{*} \circ\left(L^{-1}\right)^{\top},
$$

as easily checked, taking $U:=V:=X \times Y \times X^{*} \times Y^{*}$ and $L:=B \times\left(B^{-1}\right)^{\top}$, we get that for any representative function $f$ of $F$ the function $f \circ L$ is a representative function of $F_{A}$. Applying Proposition 3.4 to $F_{A}$ we get the next result.

COROLlaRY 3.5. Let $F \in \mathfrak{M}(X \times Y), A \in L(X, Y)$ and let $f$ be a representative of $F$. Then

$$
\begin{aligned}
\operatorname{ri}(\{A x-y \mid(x, y) \in \operatorname{dom} F\}) & ={ }^{i c}\{A x-y \mid(x, y) \in \operatorname{dom} F\} \\
& ={ }^{i c}\left\{A x-y \mid(x, y) \in \operatorname{Pr}_{X \times Y}(\operatorname{dom} f)\right\} .
\end{aligned}
$$


Assume that $0 \in{ }^{i c}\{A x-y \mid(x, y) \in \operatorname{dom} F\}$. Then the multifunction $G_{A}$ whose graph is $\left\{\left(x, x^{*}\right) \in X \times X^{*} \mid \exists y^{*} \in Y^{*}:\left(x^{*}-A^{\top} y^{*}, y^{*}\right) \in F(x, A x)\right\}$ is maximal monotone. Moreover, the function $g: X \times X^{*} \rightarrow \overline{\mathbb{R}}$ given by

$$
g\left(x, x^{*}\right)=\inf \left\{f\left(x, A x, x^{*}-A^{\top} y^{*}, y^{*}\right) \mid y^{*} \in Y^{*}\right\}
$$

is a representative of $G_{A}$ and the infimum in its definition is attained for every $\left(x, x^{*}\right) \in$ $X \times X^{*}$.

An important special case is when $M \in \mathfrak{M}(X), N \in \mathfrak{M}(Y)$ and $F:=M \times N$, that is, $F(x, y):=M(x) \times N(y)$. It is easy to prove that $F$ is (maximal) monotone if $M$ and $N$ are so. Moreover, it is quite obvious that $\varphi_{F}\left(x, y, x^{*}, y^{*}\right)=\varphi_{M}\left(x, x^{*}\right)+\varphi_{N}\left(y, y^{*}\right)$.

Corollary 3.6. Let $M \in \mathfrak{M}(X), N \in \mathfrak{M}(Y), A \in L(X, Y)$, and let $f$ and $g$ be representatives for $M$ and $N$, respectively. Then

$$
\begin{aligned}
\operatorname{ri}(A(\operatorname{dom} M)-\operatorname{dom} N) & ={ }^{i c}(A(\operatorname{dom} M)-\operatorname{dom} N) \\
& ={ }^{i c}\left(A\left(\operatorname{Pr}_{X}(\operatorname{dom} f)\right)-\operatorname{Pr}_{Y}(\operatorname{dom} g)\right) .
\end{aligned}
$$

Assume that $0 \in{ }^{i c}(A(\operatorname{dom} M)-\operatorname{dom} N)$. Then the multifunction $M+A^{\top} N A$ is maximal monotone. Moreover, if

$$
h: X \times X^{*} \rightarrow \overline{\mathbb{R}}, \quad h\left(x, x^{*}\right):=\inf \left\{f\left(x, x^{*}-A^{\top} y^{*}\right)+g\left(A x, y^{*}\right) \mid y^{*} \in Y^{*}\right\},
$$

then $h$ is a representative for $M+A^{\top} N A$ and the infimum in its expression is attained.

PROOF. For $F:=M \times N$ we have that $\operatorname{dom} F=\operatorname{dom} M \times \operatorname{dom} N$, and so the interiority assumption in the preceding corollary is satisfied. Hence $G_{A}$ is maximal monotone. But

$$
\begin{aligned}
G_{A} & =\left\{\left(x, x^{*}\right) \mid \exists y^{*} \in Y^{*}: x^{*}-A^{\top} y^{*} \in M(x), y^{*} \in N(A x)\right\} \\
& =\operatorname{gph}\left(M+A^{\top} N A\right) .
\end{aligned}
$$

The first conclusion follows from the preceding corollary.

Assume that $f$ and $g$ are representatives for $M$ and $N$, respectively. It is an easy task to verify that the function $l$ defined by $l\left(x, y, x^{*}, y^{*}\right):=f\left(x, x^{*}\right)+g\left(y, y^{*}\right)$ is a representative of $F$. Applying again the preceding corollary we find that $h$ is a representative of $M+A^{\top} N A$ and the infimum in its definition is attained.

Taking $X=Y$ and $A$ to be the identity of $X$, we get the next result for the sum of multifunctions. 
COROLlaRY 3.7. Let $M, N \in \mathfrak{M}(X)$ and let $f, g$ be representatives for $M$ and $N$, respectively. Then

$$
\text { ri } \begin{aligned}
(\operatorname{dom} M-\operatorname{dom} N) & ={ }^{i c}(\operatorname{dom} M-\operatorname{dom} N) \\
& ={ }^{i c}\left(\operatorname{Pr}_{X}(\operatorname{dom} f)-\operatorname{Pr}_{X}(\operatorname{dom} g)\right) .
\end{aligned}
$$

Assume that $0 \in{ }^{i c}(\operatorname{dom} M-\operatorname{dom} N)$. Then $M+N \in \mathfrak{M}(X)$. Moreover, if $h:=f \square_{2} g$, that is, $h\left(x, x^{*}\right):=\inf \left\{f\left(x, u^{*}\right)+g\left(x, v^{*}\right) \mid u^{*}, v^{*} \in X^{*}, u^{*}+v^{*}=x^{*}\right\}$, then $h$ is a representative for $M+N, h^{*}=f^{*} \square_{1} g^{*}$ and the infima in the expressions of $h$ and $h^{*}$ are attained.

The fact that $M+N \in \mathfrak{M}(X)$ under the condition $0 \in{ }^{i c}(\operatorname{dom} M-\operatorname{dom} N)$ has been known for a long period (see [18]). Corollary 3.7 shows that $\varphi_{M} \square_{2} \varphi_{N}$ is a representative for $M+N$ when $M, N \in \mathfrak{M}(X)$ and $0 \in{ }^{i c}(\operatorname{dom} M-\operatorname{dom} N)$, and so $\varphi_{M+N} \leq \varphi_{M} \square_{2} \varphi_{N}$; applying the result for $\psi_{M}$ and $\psi_{N}$, we obtain that $\psi_{M} \square_{2} \psi_{N}$ is also a representative of $M+N$. Hence

$$
\varphi_{M+N} \leq \varphi_{M} \square_{2} \varphi_{N} \leq \psi_{M} \square_{2} \psi_{N}=\left(\varphi_{M} \square_{2} \varphi_{N}\right)^{* \top} \leq \psi_{M+N} .
$$

The fact that $A^{\top} N A$ is maximal monotone under the condition

$$
0 \in{ }^{i c}(\operatorname{dom} N-\operatorname{Im} A)
$$

is obtained by Pennanen [13] using a result for the sum; a proof using the Fitzpatrick function is given in [23] under the same condition, and by Penot [16, Theorem 14] under the condition $Y=\mathbb{R}_{+}(\operatorname{conv}(\operatorname{dom} N)-\operatorname{Im} A)$, which is equivalent to

$$
0 \in \operatorname{core}(\operatorname{dom} N-\operatorname{Im} A) \text {, }
$$

the core or algebraic interior of $\operatorname{dom} N-\operatorname{Im} A$.

In [6], Fitzpatrick formulated Problem 5.4 in the following way: If $M$ and $N$ are monotone operators, characterise $\varphi_{M+N}$. It is not clear what kind of characterisation is meant, but a natural related question is: what is the relation between $\varphi_{M+N}$ and $\varphi_{M}$, $\varphi_{N}$ ? By an example we show that in general $\varphi_{M+N}$ does not coincide with the natural candidate $\overline{\varphi_{M} \square_{2} \varphi_{N}}$ (see (3.2) and the relations above).

EXAMPLE 1. Let $X$ be a Hilbert space (identified with its topological dual), $M=\partial\left(\|\cdot\|^{2} / 2\right), N=\partial\|\cdot\|$ and $S=M+N$. Then $S=\partial\left(\|\cdot\|^{2} / 2+\|\cdot\|\right)$, so that $M, N$ and $S$ are maximal monotone. We have that $\varphi_{M}\left(x, x^{*}\right)=\left\|x+x^{*}\right\|^{2} / 4$ and $\varphi_{N}\left(x, x^{*}\right)=\|x\|+\iota_{B_{X}}\left(x^{*}\right)$ (see [16, Examples 2 and 4]), where $\iota_{B_{X}}$ is the indicator function of the closed unit ball $B_{X}$ of $X$, and so

$$
\begin{aligned}
\left(\varphi_{M} \square_{2} \varphi_{N}\right)\left(x, x^{*}\right) & =\inf \left\{\left\|x+x^{*}-u^{*}\right\|^{2} / 4+\|x\|+\iota_{B_{x}}\left(u^{*}\right) \mid u^{*} \in X\right\} \\
& = \begin{cases}\|x\| & \text { if }\left\|x+x^{*}\right\| \leq \cdot 1, \\
\|x\|+\left(\left\|x+x^{*}\right\|-1\right)^{2} / 4 & \text { if }\left\|x+x^{*}\right\|>1 .\end{cases}
\end{aligned}
$$


It is clear that $\varphi_{M} \square_{2} \varphi_{N}$ is continuous. Since $S(0)=B_{X}$ and $S(x)=\{(1+1 /\|x\|) x\}$ for $x \neq 0$, we obtain that

$$
\begin{aligned}
\varphi_{S}\left(x, x^{*}\right) \\
=\max \left\{\sup _{u^{*} \in B_{x}}\left\langle x, u^{*}\right), \sup _{u \neq 0}\left(\frac{\|u\|+1}{\|u\|}\langle x, u\rangle+\left\langle u, x^{*}\right\rangle-\frac{\|u\|+1}{\|u\|}\langle u, u\rangle\right)\right\} \\
=\max \left\{\|x\|, \sup _{i>0,\|u\|=1}\left((t+1)\langle x, u\rangle+t\left\langle u, x^{*}\right\rangle-t(t+1)\right)\right\} \\
=\max \left\{\|x\|, \sup _{t>0}\left(\left\|(t+1) x+t x^{*}\right\|-t(t+1)\right)\right\} \\
=\sup _{t \geq 0}\left(\left\|(t+1) x+t x^{*}\right\|-t(t+1)\right) .
\end{aligned}
$$

But $\left\|(t+1) x+t x^{*}\right\| \leq\|x\|+t\left\|x+x^{*}\right\|$ and so

$$
\begin{aligned}
\mu(t) & :=\left\|(t+1) x+t x^{*}\right\|-t(t+1) \leq\|x\|+t\left\|x+x^{*}\right\|-t(t+1) \\
& =: \eta(t) \quad \forall t \geq 0 .
\end{aligned}
$$

If $\left\|x+x^{*}\right\| \leq 1$, then $\eta(t) \leq\|x\|=\eta(0)=\mu(0)$, and so $\varphi_{S}\left(x, x^{*}\right)=\|x\|=$ $\left(\varphi_{M} \square_{2} \varphi_{N}\right)\left(x, x^{*}\right)$. Assume that $\left\|x+x^{*}\right\|>1$. Then there exists some $t_{0} \geq 0$ such that $\varphi_{S}\left(x, x^{*}\right)=\mu\left(t_{0}\right) \leq \eta\left(t_{0}\right) \leq \eta\left(t_{1}\right)$, where $t_{1}:=\left(\left\|x+x^{*}\right\|-1\right) / 2$. But $\eta\left(t_{1}\right)=\left(\varphi_{M} \square_{2} \varphi_{N}\right)\left(x, x^{*}\right)$. Hence, in order to have that $\varphi_{S}\left(x, x^{*}\right)=\varphi_{M} \square_{2} \varphi_{N}\left(x, x^{*}\right)$, we need that $t_{0}=t_{1}>0$ and $\left\|\left(t_{0}+1\right) x+t_{0} x^{*}\right\|=\|x\|+t_{0}\left\|x+x^{*}\right\|$. Because $X$ is a Hilbert space, the last equality is equivalent to the existence of some $\alpha \geq 0$ such that $x=\alpha\left(x+x^{*}\right)$, and so $x$ and $x^{*}$ are collinear. So, if $x$ and $x^{*}$ are not collinear then $\varphi_{S}\left(x, x^{*}\right)<\left(\varphi_{M} \square_{2} \varphi_{N}\right)\left(x, x^{*}\right)$.

\section{Looking for autoconjugate representatives}

Fitzpatrick [6] formulated his Problem 5.5 as follows (with our notation): Given a monotone operator $T$ on $X$, find a convex function $f$ on $X \times X^{*}$ such that $\mathrm{gph} T \subset$ gph $T_{f}$ and $f^{\top}=f^{*}$. For which such $f$ is $T_{f}$ maximal monotone?

First, observe that if $f^{\top}=f^{*}$ then $f \in \mathscr{H}$. Indeed, $f$ is lsc, because $f^{* \top}$ is so. Then $g_{f}=f=f^{* T} \geq c$, and so $f \in \mathscr{H}$. It follows that $T_{f}=M_{g,}=M_{f}$. By Proposition 2.3 we have that $M_{f} \in \mathfrak{M}(X)$. Hence $T_{f} \in \mathfrak{M}(X)$ for every $f \in$ $\Lambda\left(X \times X^{*}\right)$ with $f^{\top}=f^{*}$. That result answers the last part of the problem. For the first part of the problem, the answer is practically given by [16, Theorem 10], [14] and [21]. Indeed, given a monotone operator $T: X \rightrightarrows X^{*}$, there exists $M \in \mathfrak{M}(X)$ such that $\operatorname{gph} T \subset \operatorname{gph} M$. By [16, Theorem 10] or [21, Theorem 2.4] there exists 
$f \in \Gamma\left(X \times X^{*}\right)$ such that $\varphi_{M} \leq f \leq \psi_{M}$ and $f^{\top}=f^{*}$. By Propositions 2.4 and 2.6 $\operatorname{gph} T \subset \operatorname{gph} M=\operatorname{gph} M_{f}=\operatorname{gph} T_{f}$ so that any such $f$ does the job.

The existence of such a function $f$ in [16, Theorem 10] and [21, Theorem 2.4] is obtained using Zorn's lemma. Under the additional assumption on $M \in \mathfrak{M}(X)$ that aff $(\operatorname{dom} M)$ is closed, it is possible to provide a formula for this $f$, as shown in Proposition 4.2.

Before stating the next result observe that having $f \in \mathscr{F}$ (respectively $\mathscr{G}$ ) and $\alpha>0$, then the function $g:=f_{\alpha}$ defined by $g\left(x, x^{*}\right):=\alpha f\left(x, \alpha^{-1} x^{*}\right)$ is in $\mathscr{F}$ (respectively $\mathscr{G}$ ) and $M_{g}(x)=\alpha M_{f}(x)$ for every $x \in X$; moreover, $\operatorname{Pr}_{X}(\operatorname{dom} g)=$ $\operatorname{Pr}_{X}(\operatorname{dom} f)$ and $g^{*}\left(x^{*}, x\right)=\alpha f^{*}\left(\alpha^{-1} x^{*}, x\right)$ for $x \in X$ and $x^{*} \in X^{*}$. This last relation shows that $g \in \mathscr{H}$ if $f \in \mathscr{H}$. Moreover, given a nonempty monotone multifunction $M \subset X \times X^{*}$ and $\alpha>0$, the preceding calculation of the conjugate of $f_{\alpha}$ shows that $\varphi_{\alpha M}\left(x, x^{*}\right)=\alpha \varphi_{M}\left(x, \alpha^{-1} x^{*}\right), \psi_{\alpha M}\left(x, x^{*}\right)=\alpha \psi_{M}\left(x, \alpha^{-1} x^{*}\right)$ for every $\left(x, x^{*}\right) \in X \times X^{*}$. Given two monotone multifunctions $M, N \subset X \times X^{*}$ with $\operatorname{dom} M \cap \operatorname{dom} N \neq \emptyset$, since $c_{M+N}=c_{M} \square_{2} c_{N}$, as observed before, we have $\varphi_{M+N} \leq \varphi_{M} \square_{2} \varphi_{N}$. Moreover, if $f, g \in \mathscr{F}$ (respectively $f, g \in \mathscr{G}$ ) are such that $\operatorname{Pr}_{X}(\operatorname{dom} f) \cap \operatorname{Pr}_{X}(\operatorname{dom} g) \neq \emptyset$, we easily see that $k:=f \square_{2} g \in \mathscr{F}$ (respectively $h:=\bar{k} \in \mathscr{G})$ and $\operatorname{gph}\left(M_{f}+M_{g}\right) \subset \operatorname{gph} M_{k} \subset \operatorname{gph} M_{h}:$ if $\left(x, x^{*}\right) \in \operatorname{gph}\left(M_{f}+M_{g}\right)$ and if $u^{*}, v^{*} \in X^{*}$ are such that $\left(x, u^{*}\right) \in M_{f},\left(x, v^{*}\right) \in M_{g}$ and $x^{*}:=u^{*}+v^{*}$, then $f\left(x, u^{*}\right)=\left\langle x, u^{*}\right\rangle, g\left(x, v^{*}\right)=\left\langle x, v^{*}\right\rangle$ and so

$$
\begin{aligned}
\left\langle x, x^{*}\right\rangle & \leq h\left(x, x^{*}\right) \leq k\left(x, x^{*}\right) \leq f\left(x, u^{*}\right)+g\left(x, v^{*}\right) \\
& =\left\langle x, u^{*}\right\rangle+\left\langle x, v^{*}\right\rangle=\left\langle x, x^{*}\right\rangle,
\end{aligned}
$$

hence $\left(x, x^{*}\right) \in M_{k} \subset M_{h}$. In particular, if $M_{f}+M_{g}$ is maximal monotone then $M_{h}$ is maximal monotone, too (and so $h \in \mathscr{H}$ by Proposition 2.1). Taking $N=M$ will yield the following result.

PROPOSITION 4.1. Let $M \in \mathfrak{M}(X)$ and let $f, g$ be representatives of $M$. Then, for every $\alpha \in(0,1), h:=\overline{f_{\alpha} \square_{2} g_{1-\alpha}}$ is a representative of $M$, too.

Proof. By what precedes, $M_{f_{a}}=\alpha M$ and $M_{g_{1-\alpha}}=(1-\alpha) M$. Since $M(x)$ is convex for every $x \in X$, we have that $M(x)=\alpha M(x)+(1-\alpha) M(x)=M_{f_{a}}(x)+$ $M_{g_{1-\alpha}}(x) \subset M_{h}(x)$. Since $M$ is maximal monotone we obtain that $M=M_{h}$, and so $h \in \mathscr{H}$, whence $h$ is a representative of $M$.

A special choice of $f, g, \alpha$ leads to the following construction giving a general answer to the problem raised above.

Proposition 4.2. Let $M \in \mathfrak{M}(X)$ be such that aff(dom $M)$ is closed and let $f$ be a representative of $M$. Then for $f_{1}$ and $f_{2}$ given by $f_{1}\left(x, x^{*}\right):=\frac{1}{2} f\left(x, 2 x^{*}\right)$ and 
$f_{2}\left(x, x^{*}\right):=\frac{1}{2} f^{* \top}\left(x, 2 x^{*}\right)=\frac{1}{2} f^{*}\left(2 x^{*}, x\right)$, the function $h:=f_{1} \square_{2} f_{2}$ is a representative of $M$ and $h^{*}=h^{\top}$.

Proof. Let $X_{0}:=\operatorname{aff}(\operatorname{dom} M)-\bar{x}$ for some $\bar{x} \in \operatorname{dom} M$. Then $X_{0}$ is a closed linear space. By $[20$, Lemma 5.3 (b)] (or Lemma 3.1 with $Y=\{0\}$ ) we have that

$$
\operatorname{Pr}_{X}\left(\operatorname{dom} \varphi_{M}\right) \subset \bar{x}+X_{0}
$$

and so

$$
\operatorname{aff}\left(\operatorname{Pr}_{X}\left(\operatorname{dom} \varphi_{M}\right)\right)=\operatorname{aff}(\operatorname{dom} M)=\bar{x}+X_{0} .
$$

It follows that $0 \in{ }^{i c}\left(\operatorname{Pr}_{X}\left(\operatorname{dom} \varphi_{M}\right)-\operatorname{Pr}_{X}\left(\operatorname{dom} \varphi_{M}\right)\right)$, whence, by Corollary 3.7,

$$
0 \in{ }^{i c}(\operatorname{dom} M-\operatorname{dom} M) \text {. }
$$

We have that $f \in \mathscr{H}$ and $M=M_{f}$. Then $M=M_{f \cdot \tau}$, too. It follows that $M_{f_{1}}(x)=M_{f_{2}}(x)=\frac{1}{2} M(x)$ for every $x \in X$. Moreover, by Corollary 3.7, $0 \in$ ${ }^{i c}\left(\operatorname{Pr}_{X}\left(\operatorname{dom} f_{1}\right)-\operatorname{Pr}_{X}\left(\operatorname{dom} f_{2}\right)\right), h \in \mathscr{H}$ and $M_{h}(x)=M_{f_{1}}(x)+M_{f_{2}}(x)=M(x)$ for every $x \in X$, and so $M=M_{h}$. Again using Corollary 3.7 we obtain that

$$
h\left(x, x^{*}\right)=\min \left\{\frac{1}{2} f\left(x, 2 x_{1}^{*}\right)+\frac{1}{2} f^{*}\left(2 x_{2}^{*}, x\right) \mid x^{*}=x_{1}^{*}+x_{2}^{*}\right\}=h^{*}\left(x^{*}, x\right)
$$

for all $\left(x, x^{*}\right) \in X \times X^{*}$. The proof is complete.

The preceding result suggests that we introduce a new representation of maximal monotone operators. We call it the balanced representation. For $M \in \mathfrak{M}(X)$ it is given by

$$
\beta_{M}:=\varphi_{M / 2} \bar{\square}_{2} \psi_{M / 2}:=\overline{\varphi_{M / 2} \square_{2} \psi_{M / 2}}
$$

where $M / 2$ denotes the operator given by $(M / 2)(x)=\frac{1}{2} M(x)$ for $x \in X$. Proposition 4.1 shows that $\beta_{M}$ is a representative of $M$. If $M \in \mathfrak{M}(X)$ is such that aff(dom $M$ ) is closed, then by Proposition 4.2

$$
\varphi_{M} \leq \beta_{M} \leq \psi_{M}, \quad \beta_{M}^{* \top}=\beta_{M} .
$$

What precedes show that under qualification conditions such a representative has good behaviour with respect to the usual operations.

In this paper we answered some of the questions raised by Fitzpatrick [6], at least partially. We close the paper with some questions which complete those raised by Fitzpatrick:

(1) What are the images under the mappings $\varphi$ and $\psi$ of the set $\mathscr{M}(X)$ of monotone operators? 
(2) What are the images under the mappings $T$ and $M$ of the set $\mathscr{G}$ ?

(3) What about the compositions $T \circ \varphi, M \circ \varphi, \varphi \circ T, \varphi \circ M$ ? Idem with $\varphi$ changed into $\psi$ ?

\section{References}

[1] V. Barbu, Nonlinear semigroups and differential equations in Banach spaces (Noordhoff, Leyden, 1976).

[2] H. Brezis, Opérateurs maximaux monotones et semi-groupes de contractions dans les espaces de Hilbert, North-Holland Mathematics Studies 5 (North-Holland, Amsterdam, 1973).

[3] F. E. Browder, Nonlinear operators and nonlinear equations of evolution in Banach spaces Proc. Symp. Pure Math. Vol. XVIII, Part 2 (American Mathematical Society, Providence, RI, 1976).

[4] R. S. Burachik and B. F. Svaiter, "Maximal monotone operators, convex functions and a special family of enlargements", Set-Valued Anal. 10 (2002) 297-316.

[5] R. S. Burachik and B. F. Svaiter, "Maximal monotonicity, conjugation and the duality product", Proc. Amer. Math. Soc. 131 (2003) 2379-2383.

[6] S. Fitzpatrick, "Representing monotone operators by convex functions", in Workshop and Miniconference on Functional Analysis and Optimization (Canberra, 1988), (Austral. Nat. Univ., Canberra, 1988) 59-65.

[7] D. Kinderlehrer and G. Stampacchia, An introduction to variational inequalities and their applications, Classics in Appl. Math. 31 (SIAM, Philadelphia, 2000).

[8] E. Krauss, "A representation of arbitrary maximal monotone operators via subgradients of skewsymmetric saddle functions", Nonlinear Anal. Theory Methods Appl. 9 (1985) 1381-1399.

[9] E. Krauss, "A representation of maximal monotone operators by saddle functions", Rev. Roum. Math. Pures Appl. 30 (1985) 823-836.

[10] J.-L. Lions, Quelques méthodes de résolution des problèmes aux limites non linéaires (DunodGauthier-Villars, Paris, 1969).

[11] J. E. Martínez-Legaz and B. Svaiter, "Monotone operators representable by I.s.c. functions", SetValued Anal. 13 (2005) 21-46.

[12] J. E. Martínez-Legaz and M. Théra, "A convex representation of maximal monotone operators", J. Nonlinear Convex Anal. 2 (2001) 243-247.

[13] T. Pennanen, "Dualization of generalized equations of maximal monotone type", SIAM J. Optim. $10(2000)$ 809-835.

[14] J.-P. Penot, "Autoconjugate functions and representations of monotone operators", Bull. Austral. Math. Soc. 67 (2003) 277-284.

[15] J.-P. Penot, "Is convexity useful for the study of monotonicity?", in Nonlinear Analysis and Applications (eds. R. P. Agarwal and D. O'Regan), (Kluwer, Dordrecht, 2003) 807-822.

[16] J.-P. Penot, "The relevance of convex analysis for the study of monotonicity", Nonlinear Anal. Theory Methods Appl. 58 (2004) 855-871.

[17] J.-P. Penot and C. Zæalinescu, "On the convergence of maximal monotone operators", Proc. Amer. Math. Soc. (to appear).

[18] S. Simons, Minimax and monotonicity, Lecture Notes in Mathematics 1693 (Springer, New York, 1998).

[19] S. Simons and C. Zălinescu, "A new proof for Rockafellar's characterization of maximal monotone operators", Proc. Amer. Math. Soc. 132 (2004) 2969-2972.

[20] S. Simons and C. Zălinescu, "Fenchel duality, Fitzpatrick functions and maximal monotonicity", J. Nonlinear Convex Anal. 6 (2005) 1-22. 
[21] B. F. Svaiter, "Fixed points in the family of convex representations of a maximal, monotone operator", Proc. Amer. Math. Soc. 131 (2003) 3851-3859.

[22] C. Zalinescu, Convex analysis in general vector spaces (World Scientific, Singapore, 2002).

[23] C. Złlinescu, "A new proof of the maximal monotonicity of the sum using the Fitzpatrick function", in Variational Analysis and Applications (eds. F. Giannessi and A. Maugeri), (Springer, Berlin, 2005) (to appear).

[24] E. Zeidler, Nonlinear functional analysis and its applications. II/B: Nonlinear monotone operators (Springer, New York, 1990). 